\title{
REVIEW ON TASK SCHEDULING IN UBIQUITOUS CLOUDS
}

\author{
Dr. Dinesh Kumar, \\ Assistant Professor, \\ Department of Computer Science and Engineering, \\ Koneru Lakshmaiah Education Foundation, \\ Vaddeswaram, Guntur, Andhra Pradesh, India. \\ Email: dineshngpit@gmail.com
}

\begin{abstract}
The cloud being a prominent area for research, is ubiquitous as it serves the client needs irrespective of time and place. It has become the most preferred network due to its guaranteed service rendering and cost effectiveness. The increase in the capability of the cloud services has increased the number of users to adapt to cloud. The increase in the adaption towards cloud in turn results with insufficiency in the proper and the simultaneous allocation of the resources according to the requisitions. So the task scheduling and resource allocation for the cloud becomes essential. As the conventional methods of task scheduling arrive to a local optima solution that are less-effective, the paper surveys the meta-heuristic optimization based task scheduling and the resource allocation for the ubiquitous cloud environment, that arrives to an more optimal solution at a faster rate and at ease. The paper presents the survey of the optimization techniques of task scheduling available for the cloud and the discussion of the improvement of the performance metrics in terms of make-span, throughput, cost, latency and successful service provisioning compared to the other conventional methods.
\end{abstract}

Keywords: Ubiquitous Clouds, Task scheduling, Optimization methods, guaranteed servicing and Optimized performance

\section{Introduction}

Cloud computing evolving as convenient paradigm, is gaining popularity in the day to-day life due to it's anytime, anywhere service provisioning and pay as you use method. This any time service provisioning of the cloud has increased the demand for the servicing of the cloud. So the cloud is attaining a monstrous growth as being applied in wide area of application [21] such as education, medical industry, smart city development, personal networks and so on governing all the private and the public sectors [2]. More over the anywhere service of the cloud has made it ubiquitous and most prominent. The cloud providing a ubiquitous servicing for its clients enables the users to enjoy the services without the need for the investments or the maintenance cost. Such scalable and the efficient servicing provisioning has attracted innumerable concerns into the cloud increasing the challenges of the cloud. The innumerable users adapting to cloud would increase the flow of the task requisitions which would lead to more challenges in the allocation of the resources to the service [16]. This paves to the efficient task scheduling strategies for concurrent service rendering for the cloud environment that is ubiquitous, [15] scheduling is an energy - 
Journal of ISMAC (2019)

Vol.01/ No. 01, June 2019, pp: 72-80

DOI: https://doi.org/10.36548/jismac.2019.1.006

ISSN: 2582-1369 (online)

consuming task and task allocation that are energy- efficient [2] and LWRT scheduling [3] were identified to have an energy-efficiency in task-scheduling at the cost of make-span and the throughput. The above mentioned methods were proved to be less-efficient as they arrive at to local optima solutions for the allocation of the resources. So there arises a need for the

So the paper includes the survey on the meta-heuristic optimization methods for the mapping of the works to the cloud resources [20] along with the improvement in the performance metrics of the cloud servicing. The optimization methods [12] of the cloud computing are more preferred as they arrive to more optimized solutions at increased speed at ease, causing a considerable reduction in the make span, throughput, cost, latency and the successful service provisioning.

The paper proceeds with the 2 . The survey on the optimization methods for the task-scheduling and the allocation of resources of the ubiquitous cloud computing, 3 the other methodologies available, 4 the discussion of the performance improvement achieved through the optimization techniques and the 5 the conclusion.

\section{Survey on the Meta-heuristic Optimization Methods for Task-Scheduling of Ubiquitous Cloud}

The paper on this sections elaborates the survey on the optimization methods employed for the task scheduling and the resource allocation of the ubiquitous cloud, for effective scheduling and allocation of resources. The task scheduling termed as the NP-hard problem [14] as it is more challenging and tedious. The optimization techniques arrive at a global optimum [21] at a faster and easier way than the other conventional methods. Some of the optimization methods surveyed are presented below.

Particle Swarm Optimization method and BPSO [16] and [6] uses the concepts from the social-behavior of the flocks of the birds and the school of fishes for finding the closest optimized solutions for the process of scheduling and the allocation of the resources by minimizing the response time and the computation time and maximizing the throughput of the system thus offering a successful simultaneous service provisioning.

Whale optimization method [13] the inspiration and the hunting mechanism of the whale based on the encircling, exploration and the exploitation is utilized for arriving to a most optimal method of the task scheduling and the resource allocation involving the dynamic voltage and frequency scaling. To reduce the power utilization and the 
span of the scheduling. The best solution attained ensures enhanced performance in terms of operational cost and efficiency in the service rendering.

Ant colony optimization [17] the food hunting behavior of the ant is utilized in the having the accurate solution for the NP-hard problem and the quality of the solution acquired using two objective functions that relies on the performance and the cost, to improve the task allocation on the grounds of the resource usage, cost and make-span.

Bacterial foraging [22] the solution for the scheduling is acquired employing the intelligent bacterial foraging to improve the performance and the assured servicing rendering for the cloud reducing the overall making time and reducing the complexity and the energy-utilization.

Bat algorithm [19] the bat algorithm is incorporated with the greedy approach for the work-flow scheduling of the cloud services, thus improving the multi-objective functions such as energy, time and cost, further the bat algorithm that is proved to be robust enhances the reliableness in the rendering of the services.

Chaotic optimization strategy [14] the relationship and the interactions based on the mutual, communal and the parasitic nature of the organisms along with the chaotic local search are engaged in identifying the global optimum by enhancing the population diversity and the global convergence.

Hybridized artificial bee colony optimization [4] the artificial bee colony optimization that is based on the foraging behavior of the bee is incorporated with the genetic algorithm and along with the DVFS to arrive at a global optimum for the work-flow scheduling of the ubiquitous cloud service with the simultaneous service rendering thus reducing the average execution time, cost and the energy consumption.

GA based scheduling [15] the genetic based scheduling traversing with the cross over and the mutation and converging at the global optimum is used for the task scheduling and resource allocation to identify the best allocation with the minimum time complexity and the cost.

Table 1 Survey on the Meta-Heuristic Optimization Methods

\begin{tabular}{|c|l|l|l|l|}
\hline \multicolumn{1}{|c|}{ Articles } & \multicolumn{1}{|c|}{$\begin{array}{c}\text { Optimization } \\
\text { Methods }\end{array}$} & \multicolumn{1}{c|}{ Concept } & \multicolumn{1}{c|}{ Steps Involved } & \multicolumn{1}{c|}{$\begin{array}{c}\text { Performance } \\
\text { Improvement Gained }\end{array}$} \\
\hline Alkayal et al[16] & $\begin{array}{l}\text { Particle Swarm } \\
\text { Optimization }\end{array}$ & $\begin{array}{l}\text { The foraging } \\
\text { behavior of the } \\
\text { birds }\end{array}$ & $\begin{array}{l}\text { (i)Parameter } \\
\text { initialization } \\
\text { (ii) Velocity and } \\
\text { position update. }\end{array}$ & $\begin{array}{l}\text { Make-span, } \\
\text { Energy consumption, } \\
\text { Cost-effectiveness }\end{array}$ \\
\hline
\end{tabular}




\begin{tabular}{|c|c|c|c|c|}
\hline & & & $\begin{array}{l}\text { (iii) Enumerate } \\
\text { Fitness } \\
\text { (iv) Update } \mathrm{P}_{\text {best }} \text { and } \\
\mathrm{G}_{\text {best }} \\
\text { (v) Arrange based on } \\
\text { the updated position. }\end{array}$ & \\
\hline Peng et al [13] & Whale Optimization & $\begin{array}{l}\text { Foraging behavior } \\
\text { of the whale }\end{array}$ & $\begin{array}{l}\text { (i)Encircling, } \\
\text { (ii) Exploration and } \\
\text { (iii) Exploitation }\end{array}$ & $\begin{array}{l}\text { Robust ness } \\
\text { Energy consumption } \\
\text { Throughput }\end{array}$ \\
\hline Zuo et al [17] & $\begin{array}{l}\text { Ant Colony } \\
\text { Optimization }\end{array}$ & $\begin{array}{l}\text { Food hunting } \\
\text { manners of Ant. }\end{array}$ & $\begin{array}{l}\text { (i)Initialize random } \\
\text { walk, } \\
\text { (ii)Pheromone update } \\
\text { (iii) Evaluate trail } \\
\text { based on the } \\
\text { pheromone update. } \\
\text { (iv) update global } \\
\text { trail }\end{array}$ & $\begin{array}{l}\text { Make span } \\
\text { Energy consumption. } \\
\text { Throughput } \\
\text { Cost effectiveness }\end{array}$ \\
\hline Vishrutha et al [22] & Bacterial Foraging & $\begin{array}{l}\text { Foraging behavior } \\
\text { of the bacteria }\end{array}$ & $\begin{array}{l}\text { (i)chemo taxis } \\
\text { (ii) swarming } \\
\text { (iii)reproduction } \\
\text { (iv) eliminational- } \\
\text { dispersal }\end{array}$ & $\begin{array}{l}\text { Make-span, } \\
\text { Energy consumption, } \\
\text { Throughput }\end{array}$ \\
\hline Raj et al [19] & Bat Algorithm & $\begin{array}{l}\text { Echolocation } \\
\text { behavior of the Bat }\end{array}$ & $\begin{array}{l}\text { (i)Random Walk } \\
\text { (ii) position and } \\
\text { velocity initialization } \\
\text { (iii) Position and } \\
\text { velocity update. } \\
\text { (iv) evaluate fitness } \\
\text { (v) Update global } \\
\text { near best solutions. }\end{array}$ & $\begin{array}{l}\text { Make span } \\
\text { Energy consumption } \\
\text { Cost } \\
\text { Throughput }\end{array}$ \\
\hline Abdullah et al[14] & $\begin{array}{l}\text { Chaotic } \\
\text { Optimization } \\
\text { strategy }\end{array}$ & $\begin{array}{l}\text { Relationship } \\
\text { maintenance } \\
\text { between organisms }\end{array}$ & $\begin{array}{l}\text { (i) Mutualism } \\
\text { (ii)communalism } \\
\text { (iii)parasitic nature }\end{array}$ & $\begin{array}{l}\text { Make span } \\
\text { Energy consumption } \\
\text { Cost }\end{array}$ \\
\hline Kumar et al[4] & $\begin{array}{l}\text { Artificial Bee } \\
\text { Colony } \\
\text { Optimization }\end{array}$ & $\begin{array}{l}\text { Foraging behavior } \\
\text { of the Ant bee } \\
\text { colony }\end{array}$ & $\begin{array}{l}\text { (i)random movement } \\
\text { of employed bees } \\
\text { based on memory } \\
\text { (ii) update the } \\
\text { memory } \\
\text { (iii)update on looker } \\
\text { bees } \\
\text { (iv) update scout bees } \\
\text { (v) update memory } \\
\text { with the global } \\
\text { optimum }\end{array}$ & $\begin{array}{l}\text { Energy consumption } \\
\text { Cost } \\
\text { Throughput }\end{array}$ \\
\hline Rekha et al [15] & Genetic Algorithm & $\begin{array}{l}\text { Evaluation of the } \\
\text { fittest individual }\end{array}$ & $\begin{array}{l}\text { (i)random } \\
\text { initialization } \\
\text { (ii)selection } \\
\text { (iii)cross-over } \\
\text { (iv)mutation } \\
\text { (v) fitness }\end{array}$ & $\begin{array}{l}\text { Make span } \\
\text { Throughput } \\
\text { Cost } \\
\text { Energy consumption }\end{array}$ \\
\hline
\end{tabular}




\begin{tabular}{|l|l|l|l|l|}
\hline & & enumeration & \\
\hline
\end{tabular}

The table 1 presents the meta-heuristics optimization methods surveyed and the performance improvements it provides when employed for the scheduling of the tasks in the cloud ubiquitous service provisioning.

\section{Other Methods of Task Scheduling}

The paper also includes conventional methods used in the allocation of the resources. Where Pop, et al [1] presents the adaptive method for the distributing of the service among the resources enabling a proper resource management and task scheduling, at an increased computation time and energy utilization. Measures to have efficient scheduling of the task with the energy optimization was proceeded by the Luo et al [2] by cluster framing and resulted with the increase in the make span causing time complexities. The further researches proceeded with the optimization of time as the cloud based service requisition are often time or deadline constraint admitting the EDLF and LSTR by Salehan, et al [3] offered a service rendering with the improved time complexities, better throughput but caused more energy consumption and cost. Lakshmi et al [5] optimized capacitor scheduling caused the cloud tasks to have effective scheduling that resulted with more computational-time, energy consumption and cost, the model offered a better performance in-terms of throughput but was incompatible with the time constrained service requisitions as they cause much time-complexities. Juarez, et al [11] applying the multi-heuristic rules for the scheduling of the task based on the time taken for the process, the number of the successors and the setup time enabling a parallel execution of the task showing considerable enhancement in the consumption of the energy, time and cost. The parallel execution for the large-volume of tasks results in heavy traffic causing a congestion overhead. The server power efficiency model proposed by Lin, et al [12] proffers a heuristics model based on the server efficiency and concentrates only on the power consumption of the scheduling for the tasks. The evaluation of the model shows a considerable improvement in the consumption of the energy than the other algorithms like Min-Max, but ends up with the complexities in time along with the increased cost as most of the cloud service are charged based on the time. The above details gathered based on the survey shows that the conventional optimization and heuristic methods improve the quality of the one objective function at the cost of the other. Thus limiting the scalable, reliable and the elastic services of the cloud environment.

\section{Discussion on the Performance Improvement through the Meta-heuristic Optimization}

From the paltry amount of information's gathered based researches surveyed for the metaheuristic methods of objective function optimization in the scheduling of the tasks. The performance improvement in the cloud service provisioning are discussed. The prominent cloud service handling huge amount of service has monstrous data flow 
Journal of ISMAC (2019)

Vol.01/ No. 01, June 2019, pp: 72-80

DOI: https://doi.org/10.36548/jismac.2019.1.006

ISSN: 2582-1369 (online)

in it each and every minute, the conventional methods being incompatible for the scheduling of the services as the compensate one function at the cost of the other, there arises the necessity for the survey of the most suitable mechanisms for the cloud work scheduling that is cost effective and less energy consuming. This paved way for the survey of the meta-heuristic optimization techniques available for the scheduling of the cloud services, the survey shows the metaheuristic methods based on the foraging behavior of the birds, insects animals and fishes show a quite remarkable enhancements in the computational time complexities, energy consumption, cost, throughput, and the successful service provisioning. Thus improving the efficiency of the cloud by enabling it to provide a guaranteed services even at times of the heightened service requisitions. The table 2 below shows the time complexity and the delay complexities of the conventional methods and the metaheuristic methods.

\begin{tabular}{|l|c|c|c|c|c|c|}
\hline & PSO & ACO & BAT & ABC & GA & CONV \\
\hline $\begin{array}{l}\text { Delay } \\
\text { Complexities }\end{array}$ & 0.4536 & 0.3467 & 0.4532 & 0.4723 & 0.3461 & 1.5846 \\
\hline $\begin{array}{l}\text { Time } \\
\text { Complexities }\end{array}$ & 2.456 & 2.374 & 2.454 & 2.675 & 2.318 & 4.5267 \\
\hline
\end{tabular}

Table 2 Time and the Delay complexity

The table 2 gives the time complexities and the delay complexities of the conventional methods, and the metaheuristic methods based on the survey, the time complexities and the delay complexities enumerated for the maximum number of rounds are shown in the table 2 Further the graph below gives the percentage of the successful service provisioning attained in the cloud based on the improvement attained in the energy, cost and throughput by the conventional (Conv) and the metaheuristic (META) optimizations.

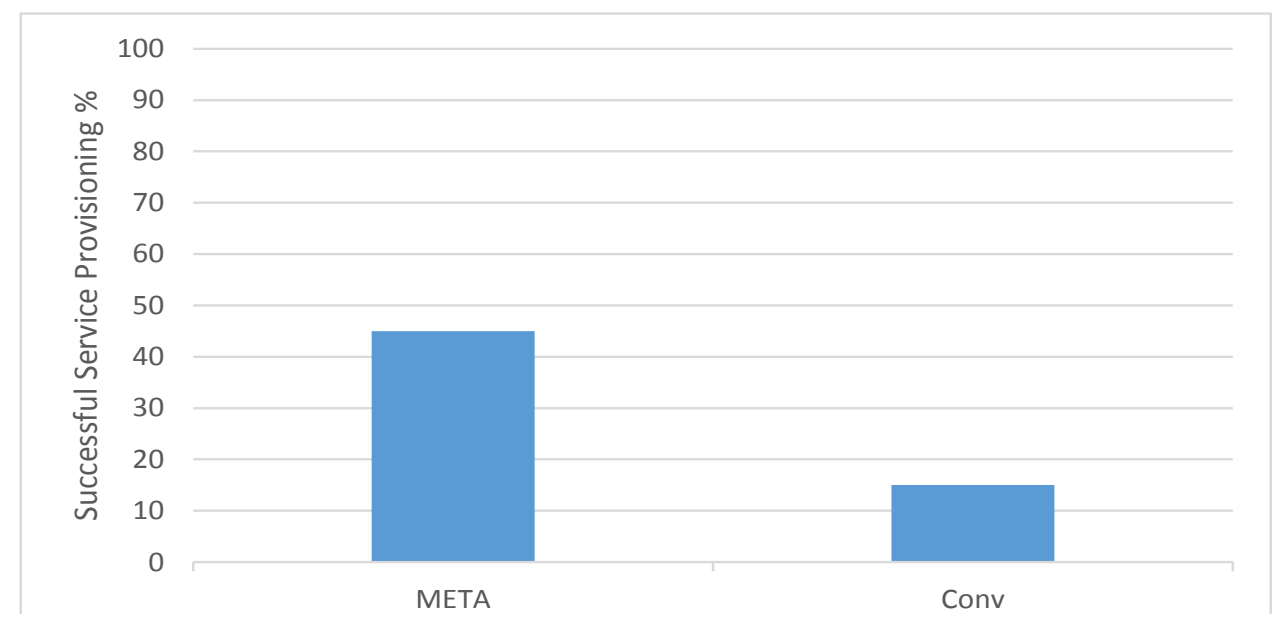


Journal of ISMAC (2019)

Vol.01/ No. 01, June 2019, pp: 72-80

DOI: https://doi.org/10.36548/jismac.2019.1.006

ISSN: 2582-1369 (online)

The fig 1 shows the successful service provisioning attained by the cloud services based on the conventional and of the metaheuristic methods, the comparison show that the meta-heuristic methods for the scheduling shows $30 \%$ of improvement than the conventional methods for the service-scheduling. The percentage of the successful service provisioning acquired from the survey conducted over the many researches for the cloud scheduling based on the conventional, and the metaheuristic methods make sure the metaheuristic methods cause a considerable improvement in the scheduling of the tasks for the cloud and the guaranteed service provisioning than the conventional methods

\section{Conclusion}

The paper reviews the details on the task scheduling for the ubiquitous cloud. The cloud being a popular service provider is prominent among a wide range application due to its quality and the guaranteed service provisioning. The wide range of applications adapting to cloud, increases the number of the service requisition to the cloud causing, difficulties in the service rendering and the timely service rendering. So the paper present the review of the task scheduling available based on both the conventional methods of optimization and the metaheuristic based optimization and from the review gathered gains the insight on the performance improvements attained by each methods. The review presented highlights that the metaheuristic methods based on the foraging behavior of the insects, reptiles, animals and the birds show remarkable improvement in the performance than the conventional methods. Further the paper is proceed with the survey on the performance improvement in the scheduling based on the hybridized meta-heuristic methods along with the authentication, improving the privacy of the service rendering.

\section{References}

[1] Pop, Florin, and Maria Potop-Butucaru. "ARMCO: Advanced topics in resource management for ubiquitous cloud computing: An adaptive approach." (2016): 79-81.

[2] Luo, Liang, Wenjun Wu, Dichen Di, Fei Zhang, Yizhou Yan, and Yaokuan Mao. "A resource scheduling algorithm of cloud computing based on energy efficient optimization methods." In 2012 International Green Computing Conference (IGCC), pp. 1-6. IEEE, 2012.

[3] Salehan, Alireza, Hossein Deldari, and Saeid Abrishami. "Performance Evaluation of Two New Lightweight Real-Time Scheduling Mechanisms for Ubiquitous and Mobile Computing Environments." Arabian Journal for Science and Engineering44, no. 4 (2019): 3083-3099.

[4] Kumar, Sunil, and Mala Kalra. "A Hybrid Approach for Energy-Efficient Task Scheduling in Cloud." In Proceedings of 2nd International Conference on Communication, Computing and Networking, pp. 10111019. Springer, Singapore, 2019.

http://irojournals.com/iroismac/ 
Journal of ISMAC (2019)

Vol.01/ No. 01, June 2019, pp: 72-80

DOI: https://doi.org/10.36548/jismac.2019.1.006

ISSN: 2582-1369 (online)

[5] Lakshmi, Adepu Sree, N. Subhash Chandra, and M. BalRaju. "Optimized Capacity Scheduler for MapReduce Applications in Cloud Environments." In Data Management, Analytics and Innovation, pp. 157-169. Springer, Singapore, 2019.

[6] Mapetu, Jean Pepe Buanga, Zhen Chen, and Lingfu Kong. "Low-time complexity and low-cost binary particle swarm optimization algorithm for task scheduling and load balancing in cloud computing." Applied Intelligence (2019): 1-23.

[7] Pop, Florin, Alexandru Iosup, and Radu Prodan. "HPS-HDS: high performance scheduling for heterogeneous distributed systems." (2018): 242-244.

[8] Muhammed, Thaha, Rashid Mehmood, Aiiad Albeshri, and Iyad Katib. "UbeHealth: a personalized ubiquitous cloud and edge-enabled networked healthcare system for smart cities." IEEE Access 6 (2018): 32258-32285.

[9] Chen, You-Shyang, Chienwen Wu, Heng-Hsing Chu, Chien-Ku Lin, and Huan-Ming Chuang. "Analysis of performance measures in cloud-based ubiquitous SaaS CRM project systems." The Journal of Supercomputing 74, no. 3 (2018): 1132-1156.

[10] Cai, Wei, Yuanfang Chi, Conghui Zhou, Chaojie Zhu, and Victor CM Leung. "UBCGaming: Ubiquitous Cloud Gaming System." IEEE Systems Journal 12, no. 3 (2018): 2483-2494.

[11] Juarez, Fredy, Jorge Ejarque, and Rosa M. Badia. "Dynamic energy-aware scheduling for parallel taskbased application in cloud computing." Future Generation Computer Systems 78 (2018): 257-271.

[12]Lin, Weiwei, Weiqi Wang, Wentai Wu, Xiongwen Pang, Bo Liu, and Ying Zhang. "A heuristic task scheduling algorithm based on server power efficiency model in cloud environments." Sustainable Computing: Informatics and Systems 20 (2018): 56-65. (scheduling impacts over processing time and energy consumption)

[13] Peng, Hua, Wu-Shao Wen, Ming-Lang Tseng, and Ling-Ling Li. "Joint optimization method for task scheduling time and energy consumption in mobile cloud computing environment." Applied Soft Computing 80 (2019): 534-545.

[14] Abdullahi, Mohammed, Md Asri Ngadi, Salihu Idi Dishing, and Barroon Isma'eel Ahmad. "An efficient symbiotic organism's search algorithm with chaotic optimization strategy for multi-objective task scheduling problems in cloud computing environment." Journal of Network and Computer Applications 133 (2019): 60-74.

[15] Rekha, P. M., and M. Dakshayini. "Efficient task allocation approach using genetic algorithm for cloud environment." Cluster Computing (2019): 1-11.

[16] Alkayal, Entisar S., Nicholas R. Jennings, and Maysoon F. Abulkhair. "Efficient task scheduling multiobjective particle swarm optimization in cloud computing." In 2016 IEEE 41st Conference on Local Computer Networks Workshops (LCN Workshops), pp. 17-24. IEEE, 2016.

[17]Zuo, Liyun, Lei Shu, Shoubin Dong, Chunsheng Zhu, and Takahiro Hara. "A multi-objective optimization scheduling method based on the ant colony algorithm in cloud computing." Ieee Access 3 (2015): 26872699. 
[18] Dai, Yangyang, Yuansheng Lou, and Xin Lu. "A task scheduling algorithm based on genetic algorithm and ant colony optimization algorithm with multi-QoS constraints in cloud computing." In 2015 7th International Conference on Intelligent Human-Machine Systems and Cybernetics, vol. 2, pp. 428-431. IEEE, 2015.

[19] Raj, Bibhav, Pratyush Ranjan, Naela Rizvi, Prashant Pranav, and Sanchita Paul. "Improvised Bat Algorithm for Load Balancing-Based Task Scheduling." In Progress in Intelligent Computing Techniques: Theory, Practice, and Applications, pp. 521-530. Springer, Singapore, 2018.

[20] Agrawal, Mayur, Rishabh Bansal, Ankur Choudhary, and Arun Prakash Agrawal. "Hetrogenous Computing Task Scheduling Using Improved Harmony Search Optimization." In 2018 International Conference on Advances in Computing, Communication Control and Networking (ICACCCN), pp. 11-15. IEEE, 2018.

[21] Kilıç, Haydar, and Uğur Yüzgeç. "Improved antlion optimization algorithm via tournament selection and its application to parallel machine scheduling." Computers \& Industrial Engineering 132 (2019): 166-186.

[22] Vishrutha, T., and P. Chitra. "Efficient Task Allocation Using Intelligent Bacterial Foraging Optimization (IBFO) Algorithm in Cloud." In 2019 1st International Conference on Innovations in Information and Communication Technology (ICIICT), pp. 1-5. IEEE, 2019. 